\title{
LOCATION AND STABILITY OF THE TRIANGULAR POINTS IN THE TRIAXIAL ELLIPTIC RESTRICTED THREE-BODY PROBLEM
}

\author{
M. Radwan ${ }^{1}$ and Nihad. S. Abd El Motelp ${ }^{2}$ \\ Received February 4 2021; accepted April 122021
}

\begin{abstract}
The main goal of the present paper is to evaluate the perturbed locations and investigate the linear stability of the triangular points. We studied the problem in the elliptic restricted three body problem frame of work. The problem is generalized in the sense that the two primaries are considered as triaxial bodies. It was found that the locations of these points are affected by the triaxiality coefficients of the primaries and the eccentricity of orbits. Also, the stability regions depend on the involved perturbations. We also studied the periodic orbits in the vicinity of the triangular points.
\end{abstract}

\section{RESUMEN}

Nuestro objetvo principal es determinar las ubicaciones perturbadas e investigar la estabilidad lineal de los puntos triangulares. Realizamos nuestro estudio en el marco del problema elíptico restringido de tres cuerpos. Generalizamos el problema considerando a los dos cuerpos primarios como cuerpos triaxiales. Encontramos que la ubicación de los puntos triangulares se ve afectada por los coeficientes de triaxialidad de los cuerpos primarios y por la excentricidad de las órbitas. También notamos que las regiones de estabilidad dependen de las perturbaciones inducidas y estudiamos las órbitas periódicas en la vecindad de los puntos triangulares.

Key Words: celestial mechanics - gravitation - methods: analytical — methods: numerical — planets and satellites: dynamical evolution and stability

\section{INTRODUCTION}

The restricted three-body problem (R3BP) is the most significant problem in celestial mechanics. This due to its wide applications in space dynamics and solar system dynamics. The knowledge of the R3BP is crucial in almost all space applications. We can define this problem as a dynamical system in which an infinitesimal body moves in the gravitational field of two massive bodies (primaries). The two primaries move about their common centre of mass in either circular or elliptic orbits, which leads to the famous circular or elliptic restricted problems, respectively (Musielak \& Quarles 2014). In the elliptic restricted three-body problem, ER3BP, it is assumed that the smaller primary moves around the more massive one in an elliptical orbit. The influence of the infinitesimal mass on the motion of the primaries is negli-

\footnotetext{
${ }^{1}$ Astronomy; Space Science and Metreology Department, Faculty of Science, Cairo University, 12613 Giza, Egypt.

${ }^{2}$ Astronomy Department, National Research Institute of Astronomy and Geophysics (NRIAG), 11421 Helwan, Cairo, Egypt.
}

gible. This significantly simplified model has more applications in space dynamics than the more general problem.

Despite the fact that the RTBP is not integrable, five specified solutions in the rotating frame are known. These solutions correspond to equilibrium positions. Three of these equilibrium points, known as collinear points, are located on the line joining the two massive primaries in the rotating reference frame. The remaining two points, called triangular points, form equilateral triangles with the massive primaries. The determination of the location of these libration points is of great interest for different space applications (Abd El-Salam 2019). In general the equilateral equilibrium points are conditionally stable, while the collinear libration points are usually unstable (Singh \& Tyokyaa 2016).

Most of the celestial bodies are of irregular nature and cannot be assumed to be spherical in the RTBP, because their stability of movement is influenced by their shape. The planets of the solar system and 
their satellites are assumed as extended bodies and cannot be regarded as spheres. Furthermore, the effect of eccentricity on the orbits is significant. Hence, in order to achieve an acceptable accuracy, we have to take into account that the orbits of most celestial bodies are elliptic rather than circular (Singh \& Tyokyaa 2016).

From the theory of small oscillations around the triangular points two types of periodic orbits can be obtained; short-period orbits with periods approximately the same as the orbital period of the primaries, and long-period orbits with periods many times that of the primaries. The importance of investigating these periodic orbits lies in their frequent appearance in nature; they provide us with important information about orbital resonances, spin orbits and are also useful to approximate quasi-periodic trajectories (Abouelmagd (2013), Mittal et al. (2009), Kumar \& Sharma (2020), Ahmed et al. (2020)).

A number of studies have been performed on the equilateral triangular points of the restricted threebody problem. Singh \& Begha (2011) investigated the existence of periodic orbits about the triangular points, namely $L_{4}$ and $L_{5}$, in the RTBP when the primaries are considered as triaxial and oblate spheroid bodies, besides perturbations due to Coriolis and centrifugal forces. The authors found that long and short periodic orbits exist around these points, and that their eccentricities and periods are influenced by the involved perturbations. Singh \& Umar (2012) studied the stability of triangular points in the ER3BP, when both oblate primaries emit light energy. The locations of $L_{4}$ and $L_{5}$ are shifted away from the line joining the two primaries, as compared to the classical case. The stability region decreases or increases with variations in eccentricity, oblateness and radiation pressure. Naryan \& Usha (2014) investigated the stability of infinitesimal motions around the equilateral points. They considered the more massive primary as a radiant source and the less massive one as a triaxial body. Zahra et al. (2016), Zahra et al. (2017) studied the locations and linear stability of the triangular points under the effects of oblateness and triaxiality of the primaries, plus small relativistic perturbations. They found that the locations of the triangular points are affected by the considered perturbations. Numerical explorations were carried out to show the effect of perturbations on the position and stability. Singh \& Tyokyaa (2016) investigated the positions and stability of the libration points when both primaries are taken as oblate spheroids with oblateness up to the fourth zonal harmonic $J_{4}$. They found that both har- monic coefficients $J_{2}$ and $J_{4}$, eccentricity and semimajor axis have destabilizing tendencies and as a result the size of the region of stability decreases with an increase in the parameters involved.

Recently, Hadia et al. (2019) studied analytically the existence and the linear stability of the libration points assuming the primaries as triaxial bodies and the Euler angles as specified values. The authors proved that the positions and the stability of the triangular points change according to the effect of the triaxiality of the primaries. Furthermore, they presented the solution of long and short periodic orbits for a stable motion. Cárcamo-Díaz et al. (2020) revisited the circular spatial restricted problem. The authors presented some new results in the light of the concept of Lie stability. This paper studies the positions and stability of the well-known triangular points, in the elliptic restricted problem frame of work. The triaxiality of the primary bodies is taken into consideration. In addition, we study the periodic orbits around the triangular points. To examine the present problem we carry out several numerical explorations.

\section{DYNAMICAL MODEL}

Let $m_{1}, m_{2}$ and $m_{3}$ be the masses of the primary, secondary, and the third infinitesimal body, respectively. The third body moves under the effect of the triaxial primaries, but it does not affect their motion. The two primaries move around their common centre of mass in elliptic orbits. We consider a rotating coordinate system $(x, y, z)$ with origin at the centre of mass of the primaries. The primaries are permanently located on the $\mathrm{x}$-axis, which is the line joining them. When investigating the restricted three-body problem it is better to choose a system of normalized units. So, we assume that the distance between the primaries is unity and we choose also the unit of time to make the gravitational constant unity. The total mass of the primaries is considered as 1, i.e. $m_{1}+m_{2}=1$. In this normalized system we adopt $\mu=\frac{m_{2}}{m_{1}+m_{2}}$ as a dimensionless mass parameter. According to the above considerations, the equations of motion of the third infinitesimal body moving under the effect of the triaxial primaries located at $(\mu, 0),(1-\mu, 0)$ are given by (Ahmed et al. 2020)

$$
\ddot{x}-2 n \dot{y}=\frac{\partial U}{\partial x}, \quad \ddot{y}+2 n \dot{x}=\frac{\partial U}{\partial y},
$$


with

$$
\begin{gathered}
U=\frac{n^{2}}{2}\left[(1-\mu) r_{1}^{2}+\mu r_{2}^{2}\right]+\frac{(1-\mu)}{r_{1}}+\frac{\mu}{r_{2}}+ \\
\frac{(1-\mu)\left(2 \sigma_{1}-\sigma_{2}\right)}{2 r_{1}^{3}}-\frac{3(1-\mu)\left(\sigma_{1}-\sigma_{2}\right) y^{2}}{2 r_{1}^{5}}+ \\
\frac{\mu\left(2 \gamma_{1}-\gamma_{2}\right)}{2 r_{2}^{3}}-\frac{3 \mu\left(\gamma_{1}-\gamma_{2}\right) y^{2}}{2 r_{2}^{5}} . \\
r_{1}=\sqrt{(x+\mu)^{2}+y^{2}}, \\
r_{2}=\sqrt{(x+\mu-1)^{2}+y^{2}} \\
n=\frac{1}{a\left(1-e^{2}\right)}\left\{1+\frac{3}{2}\left[\left(2 \sigma_{1}-\sigma_{2}\right)+\left(2 \gamma_{1}-\gamma_{2}\right)\right]\right\}
\end{gathered}
$$

Here, $U$ is the restricted three-body potential; $n$ is the mean motion of the primaries; $r_{1}$ and $r_{2}$ are the distances of the infinitesimal mass from the primaries; $\sigma_{1}, \sigma_{2}$ and $\gamma_{1}, \gamma_{2}$ are the triaxiality coefficients of the bigger and smaller primaries, respectively.

\section{LOCATIONS OF THE TRIANGULAR POINTS}

The libration points represent stationary solutions of the restricted three-body problem. The positions of these points can be obtained by setting all components of the relative velocity and acceleration equal to zero, i.e. we found them by setting $\dot{x}=\dot{y}=0=\ddot{x}=\ddot{y}$. Consequently, the locations of these points can be found by solving simultaneously the nonlinear equations $U_{x}=U_{y}=0$, and $y \neq 0$. Setting

$$
\begin{aligned}
& A_{\gamma}=\gamma_{1}-\gamma_{2}, \quad A_{\sigma}=\sigma_{1}-\sigma_{2}, \\
& A_{\alpha}=2 \gamma_{1}-\gamma_{2}, \quad A_{\beta}=2 \sigma_{1}-\sigma_{2},
\end{aligned}
$$

we have

$$
\begin{aligned}
U_{x} & =-\mu(-1+x+\mu)\left[\frac{1}{r_{2}^{3}}+\frac{3}{2 r_{2}^{5}} A_{\alpha}-\frac{15 y^{2}}{2 r_{2}^{7}} A_{\gamma}\right] \\
& +\frac{x}{a\left(1-e^{2}\right)}+(1-\mu)(x+\mu)\left[\frac{1}{r_{1}^{3}}-\frac{3}{2 r_{1}^{5}} A_{\beta}\right. \\
& \left.+\frac{3}{2 a\left(1-e^{2}\right)}\left(A_{\alpha}+A_{\beta}\right)-\frac{15 y^{2}}{2 r_{1}^{7}} A_{\sigma}\right],
\end{aligned}
$$

$$
\begin{aligned}
& U_{y}=-y\left\{\frac{\mu}{r_{2}^{3}}+\frac{1-\mu}{r_{1}^{3}}+\frac{3 \mu}{2 r_{2}^{5}} A_{\alpha}+\frac{3(1-\mu)}{2 r_{1}^{5}} A_{\beta}-\right. \\
& \frac{1}{a\left(1-e^{2}\right)}\left[1+\frac{3}{2}\left(A_{\alpha}+A_{\beta}\right)\right]- \\
& \frac{15 y^{2}}{2}\left[\frac{\mu A_{\gamma}}{r_{2}^{7}}+\frac{(1-\mu)}{r_{1}^{7}} A_{\sigma}\right]- \\
& \left.\frac{3 \mu}{r_{2}^{5}} A_{\gamma}+\frac{3(1-\mu)}{r_{1}^{5}} A_{\sigma}\right\},
\end{aligned}
$$

Ignoring the triaxiality perturbations due to the primary bodies yields the equilateral solution of the classical restricted three-body problem i.e. $r_{1}=$ $r_{2}=1$. Then, it may be reasonable to consider that the locations of these points are the same as given by classical problem, but perturbed by small terms, i.e.

$$
r_{i}=1+\delta_{i}, \quad \delta_{i}<<1, \quad(i=1,2) .
$$

Substituting this assumption (7) into (3), solving for $x$ and $y$ and retaining terms up to the first order in the small quantities $\delta_{i}$ we get:

$$
\begin{aligned}
& x=\frac{1}{2}\left(2 \delta_{1}-2 \delta_{2}-2 \mu+1\right), \\
& y= \pm \frac{1}{2} \sqrt{\left(3+4\left(\delta_{1}+\delta_{2}\right)\right)} .
\end{aligned}
$$

Substituting the values of $r_{1}, r_{2}, x$ and $y$ into equations (5) and (6) and ignoring the terms higher than order one in $\delta_{i}$ we obtain the following two simultaneous equations in $\delta_{1}$ and $\delta_{2}$.

$$
A_{1}+A_{2} \delta_{1}+A_{3} \delta_{2}=0, \quad B_{1}+B_{2} \delta_{1}+B_{3} \delta_{2}=0 .
$$

The corresponding solution is:

$$
\begin{gathered}
\delta_{1}=-\frac{A_{3} B_{1}-A_{1} B_{3}}{A_{3} B_{2}-A_{2} B_{3}}, \quad A_{3} B_{2}-A_{2} B_{3} \neq 0, \\
\delta_{2}=-\frac{-A_{2} B_{1}+A_{1} B_{2}}{A_{3} B_{2}-A_{2} B_{3}}, \quad A_{3} B_{2}-A_{2} B_{3} \neq 0,
\end{gathered}
$$




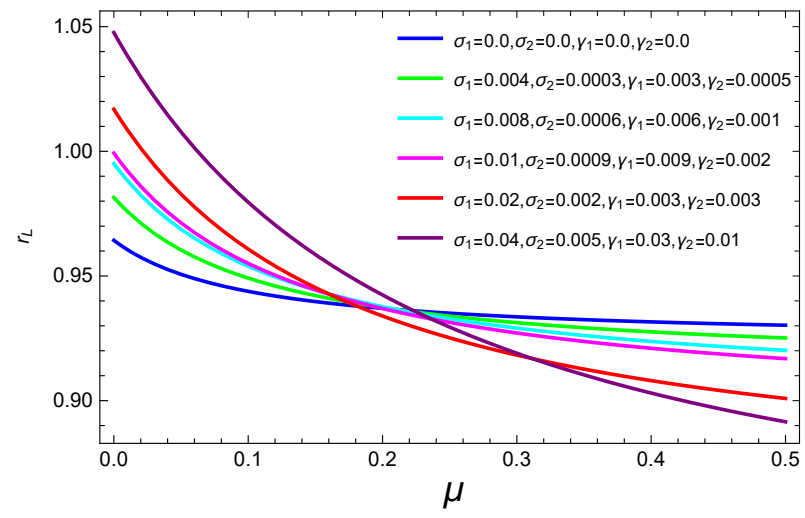

Fig. 1. The locations of $L_{4,5}$ with different triaxiality coefficients and eccentricity $e=0.2$. The color figure can be viewed online.

where:

$$
\begin{aligned}
& A_{1}=-\frac{1}{2}+\mu+\frac{3}{4}\left(\mu A_{\alpha}-A_{\beta}+\mu A_{\beta}\right)+ \\
& \frac{3}{4 a\left(1-e^{2}\right)}\left(A_{\alpha}+A_{\beta}\right)+ \\
& \frac{3}{2 a\left(1-e^{2}\right)}\left(1-\mu\left(2+3 A_{\alpha}+3 A_{\beta}\right)\right)+ \\
& \frac{45}{16}\left(-\mu\left(A_{\gamma}+A_{\sigma}\right)+A_{\sigma}\right) \text {, } \\
& A_{2}=\frac{1}{2}-\frac{1}{a\left(1-e^{2}\right)}\left(1+\frac{3}{2}\left(A_{\alpha}+A_{\beta}\right)\right)+ \\
& \frac{3}{2}\left(1-\mu A_{\alpha}+\frac{3}{2}(1-\mu) A_{\beta}-\frac{55}{8}(1-\mu) A_{\sigma}+\frac{5}{4} \mu A_{\gamma}\right) \text {, } \\
& A_{3}=1+\frac{3}{2} A_{\beta}+\frac{1}{a\left(1-e^{2}\right)}\left(1+\frac{3}{2}\left(A_{\alpha}+A_{\beta}\right)\right)- \\
& \frac{15}{8} A_{\sigma}-\frac{3}{2} \mu\left(1+\frac{3}{2} A_{\alpha}+A_{\beta}-\frac{55}{8}-\frac{5}{4} A_{\sigma}\right) \\
& \begin{array}{r}
B_{1}=-\frac{\sqrt{3}}{2}\left\{1-\frac{1}{a\left(1-e^{2}\right)}\left[1+\frac{3}{2}\left(A_{\alpha}+A_{\beta}\right)\right]-\right. \\
\left.\quad \frac{3}{2}\left[A_{\beta}-\frac{7}{4} A_{\sigma}+\mu\left(A_{\alpha}-A_{\beta}-\frac{7}{4} A_{\gamma}+\frac{7}{4} A_{\sigma}\right)\right]\right\},
\end{array}
\end{aligned}
$$

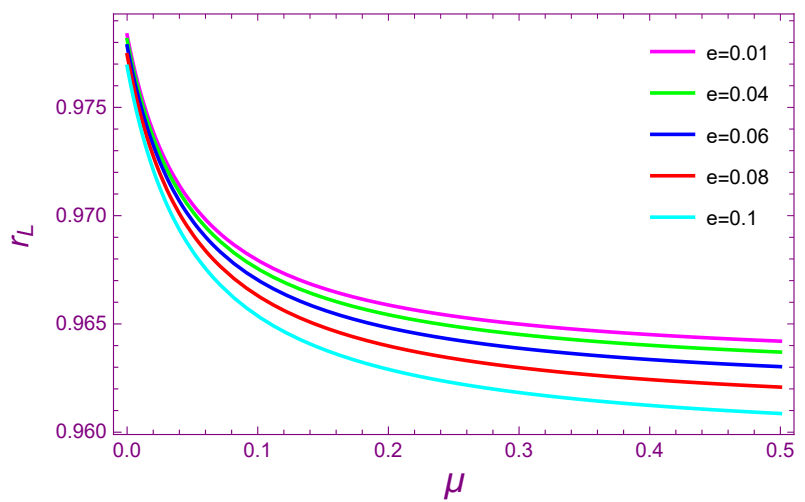

Fig. 2. The locations of $L_{4,5}$ with different eccentricities and triaxiality coefficients $\sigma_{1}=0.0002, \sigma_{2}=0.0005$, $\gamma_{1}=0.003$ and $\gamma_{2}=0.002$. The color figure can be viewed online.

$$
\begin{gathered}
B_{2}=\frac{7}{2 \sqrt{3}}+\frac{1}{\sqrt{3} a\left(1-e^{2}\right)}\left[1+\frac{3}{2}\left(A_{\alpha}+A_{\beta}\right)\right]+ \\
\frac{\sqrt{3}}{2}\left[\frac{13}{2} A_{\beta}-\frac{121}{8} A_{\sigma}-\right. \\
\left.\mu\left(3+A_{\alpha}+\frac{13}{2} A_{\beta}-\frac{37}{4} A_{\gamma}-\frac{121}{8} A_{\sigma}\right)\right] \\
B_{3}=-\frac{1}{\sqrt{3}}\left(1+\frac{1}{a\left(-1+e^{2}\right)}\right)+ \\
\frac{3 \sqrt{3}}{2}\left[\frac{37}{4} A_{\sigma}-\frac{A_{\beta}}{3}+\mu\left(1+\frac{13}{2} A_{\alpha}+\right.\right. \\
\left.\left.A_{\beta}-\frac{121}{8} A_{\gamma}-\frac{37}{4} A_{\sigma}\right)-\frac{\left(A_{\alpha}+A_{\beta}\right)}{a\left(-1+e^{2}\right)}\right]
\end{gathered}
$$

\section{GRAPHICAL REPRESENTATIONS (LOCATION)}

In Figures 1-2 we plot the locations $r_{L_{4,5}}$ of the triangular points in the elliptic restricted three body problem, taking into consideration triaxiality and eccentricity effects. Figure 1 represents the variation of $r_{4,5}$ against the mass ratio $\mu$. The calculations are carried out for several dynamical models with different coefficients $\gamma_{i}$ and $\sigma_{i}$; and constant eccentricity of the orbit of the primary $e=0.2$. As is clear from the figure, the locations begin to decrease sharply until we reach the value $\mu \approx 0.15$, then they decrease slowly. We also observe that the variation 
in the locations $r_{L_{4,5}}$ is small, because the triaxiality coefficients are very small. Figure 2 depicts the variation of $r_{L_{4,5}}$ against the mass ratio $\mu$ with constant triaxiality coefficients. We applied our case study to a range of eccentricities; the eccentricity $e$ takes the values $0.01,0.04,0.06,0.08$ and 0.1 . We notice that the change in the curves is nearly the same for all considered cases. This is because the chosen values of $e$ are small. The dynamics is approximately the same but with different sizes of the perturbations. We expect that the larger the eccentricity the larger will be the perturbation in the positions of the triangular points.

\section{STABILITY OF THE TRIANGULAR POINTS}

The dynamical models which describe the restricted problem are very difficult. The great complexity of these models leads us to focus our attention on linearized dynamics, because in this case we obtain simplified mathematical expressions that could be handled easily. The location of the third body is displaced little from the equilibrium point due to the considered perturbations. If the resultant motion of this body is a rapid departure from this point, we call such a position of equilibrium point an unstable one; if the body just oscillates about the point, it is said to be a stable position (Abd El-Salam 2019). To examine the possible motion of the infinitesimal body in the proximity of the triangular points, the equations of motion should be linearized. Let the position of the triangular points be $\left(x_{\circ}, y_{\circ}\right)$ and let the third body be displaced to the point $\left(x_{\circ}+\xi, y_{\circ}+\eta\right)$, where $\xi$ and $\eta$ are small displacement in $\left(x_{\circ}, y_{\circ}\right)$, so that the linearized equations can be written as:

$$
\begin{aligned}
& \ddot{\xi}-2 n \dot{\eta}=\frac{1}{a\left(1-e^{2}\right)}\left(U_{x x}^{L_{4,5}} \xi+U_{x y}^{L_{4,5}} \eta\right), \\
& \ddot{\eta}-2 n \dot{\xi}=\frac{1}{a\left(1-e^{2}\right)}\left(U_{y y}^{L_{4,5}} \eta+U_{x y}^{L_{4,5}} \xi\right) .
\end{aligned}
$$

Here $U_{x x}^{L_{4,5}}$ denotes the second derivative of $U$ with respect to $x$ computed at the stationary solution and the rest of the derivatives are defined similarly. The characteristic equation corresponding to equation (18) may be written as

$$
\begin{array}{r}
\lambda^{4}+\left(4 n^{2}-U_{x x}^{L_{4,5}}-U_{y y}^{L_{4,5}}\right) \lambda^{2}+ \\
U_{x x}^{L_{4,5}} U_{y y}^{L_{4,5}}-\left(U_{x y}^{L_{4,5}}\right)^{2}=0,
\end{array}
$$

which may be written in the form

$$
\lambda^{4}+U_{L_{i}} \lambda^{2}+\nu_{L_{i}}=0,
$$

with

$$
\begin{aligned}
& U_{L_{i}}=\left(4 n^{2}-U_{x x}^{L_{4,5}}-U_{y y}^{L_{4,5}}\right), \\
& \nu_{L_{i}}=U_{x x}^{L_{4,5}} U_{y y}^{L_{4,5}}-\left(U_{x y}^{L_{4,5}}\right)^{2} .
\end{aligned}
$$

The roots of equation (21) are

$$
\lambda_{1,2}= \pm \frac{\sqrt{-U_{L_{i}}-\sqrt{U_{L_{i}}^{2}-4 \nu_{L_{i}}}}}{\sqrt{2}}
$$

and

$$
\lambda_{3,4}= \pm \frac{\sqrt{-U_{L_{i}}+\sqrt{U_{L_{i}}^{2}-4 \nu_{L_{i}}}}}{\sqrt{2}} .
$$

The possible solutions for equation (19) will determine the stability of the equilibrium points. We have three possibilities: asymptotically stable, when all the solutions are real and negative; unstable, when at least one of the solutions is positive and real; and finally, the stable case when all solutions are purely imaginary (Szebehely 1967). We will focus our attention only on the case with oscillatory stable solutions about the triangular points. To investigate the stability/instability we first compute the partial derivatives required for equations (22) and (23) as

$$
\begin{aligned}
U_{x x} & =\frac{1}{a\left(1-e^{2}\right)}\left[1+\frac{3}{2}\left(A_{\alpha}+A_{\beta}\right)\right]+ \\
& \mu\left\{\frac{3(\mu+x-1)^{2}}{r_{2}^{5}}-\frac{1}{r_{2}^{3}}+\right. \\
& \frac{3}{2} A_{\alpha}\left[\frac{5(\mu+x-1)^{2}}{r_{2}^{7}}-\frac{1}{r_{2}^{5}}\right]- \\
& \left.\frac{15}{2} y^{2} A_{\gamma}\left[\frac{7}{r_{2}^{9}}(\mu+x-1)^{2}-\frac{1}{r_{2}^{7}}\right]\right\}+ \\
& (1-\mu)\left\{\frac{3}{2} A_{\beta}\left[\frac{5(\mu+x)^{2}}{r_{1}^{7}}-\frac{1}{r_{1}^{5}}\right]+\right. \\
& {\left.\left[\frac{3(\mu+x)^{2}}{r_{1}^{5}}-\frac{1}{r_{1}^{3}}\right]-\frac{15}{2} y^{2} A_{\sigma}\left[\frac{7(\mu+x)^{2}}{r_{1}^{9}}-\frac{1}{r_{1}^{7}}\right]\right\}, }
\end{aligned}
$$




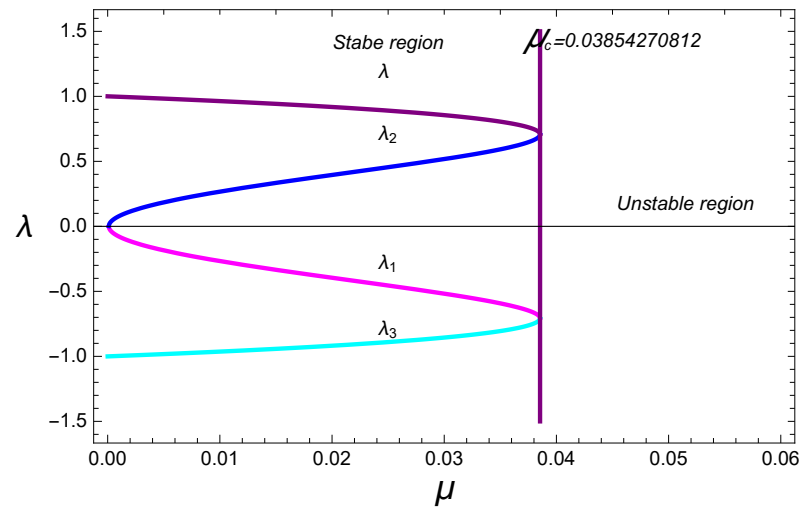

Fig. 3. The imaginary part of the eigenvalues versus the mass parameter in the classical case, $\sigma_{i}=\gamma_{i}=0$. The color figure can be viewed online.

$$
\begin{gathered}
U_{y y}=\frac{1}{a\left(1-e^{2}\right)}\left[1+\frac{3}{2}\left(A_{\alpha}+A_{\beta}\right)\right]+ \\
\mu\left\{3 A_{\gamma}\left[\frac{10 y^{2}}{r_{2}^{7}}-\frac{5 y^{2}}{2}\left(\frac{7 y^{2}}{r_{2}^{9}}-\frac{1}{r_{2}^{7}}\right)-\frac{1}{r_{2}^{5}}\right]\right\}+ \\
(1-\mu)\left\{\frac{3 y^{2}}{r_{1}^{5}}-\frac{1}{r_{1}^{3}}+\frac{3}{2}\left(\frac{5 y^{2}}{r_{1}^{7}}-\frac{1}{r_{1}^{5}}\right) A_{\beta}-\right. \\
3 A_{\sigma}\left[\frac{1}{r_{1}^{5}}+5 y^{2}\left(\frac{-2}{r_{1}^{7}}+\frac{7 y^{2}}{2 r_{1}^{9}}-\frac{1}{2 r_{1}^{7}}\right)\right]+ \\
\left.\frac{3 A_{\alpha}}{2}\left(\frac{5 y^{2}}{r_{2}^{7}}-\frac{1}{r_{2}^{5}}\right)+\frac{3 y^{2}}{r_{2}^{5}}-\frac{1}{r_{2}^{3}}\right\}, \\
U_{x y}=y\left\{\mu ( \mu + x - 1 ) \left[-\frac{105 y^{2} A_{\gamma}}{2 r_{2}^{9}}+\frac{15 A_{\alpha}}{2 r_{2}^{7}}+\right.\right. \\
\left.\frac{15 A_{\gamma}}{r_{2}^{7}}+\frac{3}{r_{2}^{5}}\right]+y(1-\mu)(\mu+x)\left[\frac{3}{r_{1}^{5}}-\right. \\
\left.\left.\frac{105 y^{2} A_{\sigma}}{2 r_{1}^{9}}+\frac{15 A_{\beta}}{2 r_{1}^{7}}+\frac{15 A_{\sigma}}{r_{1}^{7}}\right]\right\} .
\end{gathered}
$$

\section{GRAPHICAL REPRESENTATIONS (STABILITY)}

Figures 3-5 illustrate the stability regions taking into consideration the triaxiality coefficients and the eccentricity of the orbits. Figure 3 represents the classical case i.e. when $\sigma_{i}=0, \gamma_{i}=0$ and $e=0$ as well. Figure 4 depicts the stability regions for dynamical systems with different triaxiality coefficients, while

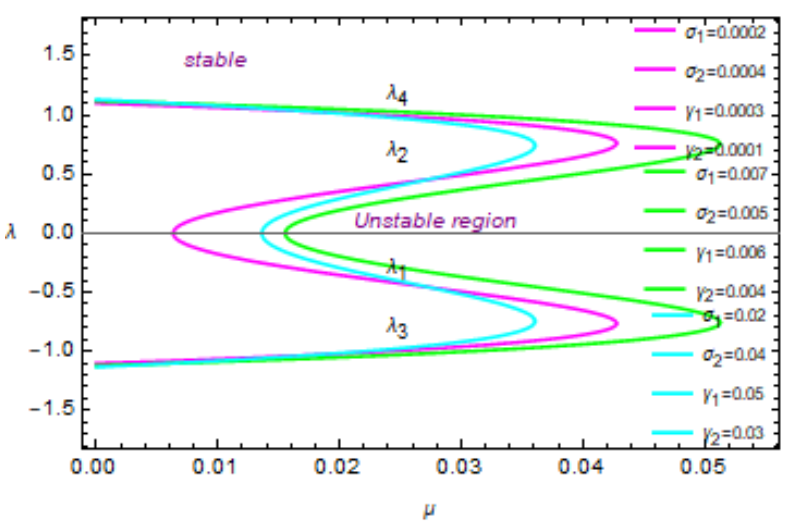

Fig. 4. The imaginary part of the eigenvalues versus the mass parameter with eccentricity $e=0.01$ and different triaxial parameters. The color figure can be viewed online.

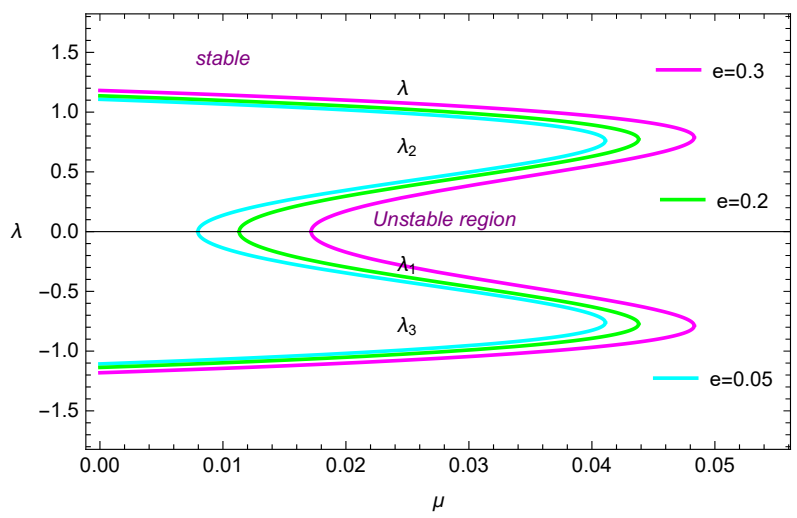

Fig. 5. The imaginary part of the eigenvalues versus the mass parameter with different eccentricities and triaxial parameters, $\sigma_{1}=0.001, \sigma_{2}=0.004, \gamma_{1}=0.003$ and $\gamma_{2}=0.002$. The color figure can be viewed online.

Figure 5 shows these regions for different eccentricities and constant coefficients. We can see from Figure 4 that each stability region is characterized by different values of the triaxiality coefficients. The size of the regions is not much different from that of the classical case; this because the perturbation due to the triaxiality coefficients is small. In Figure 5 the effect of different eccentricities on the size of the regions is obvious. Comparing with previous work e.g. Abd El-Salam (2015), we found that the size of the regions is slightly different from that found in the quoted paper due to the perturbations considered in each case. We expect that as the eccentricity increases the stability regions will be de- 


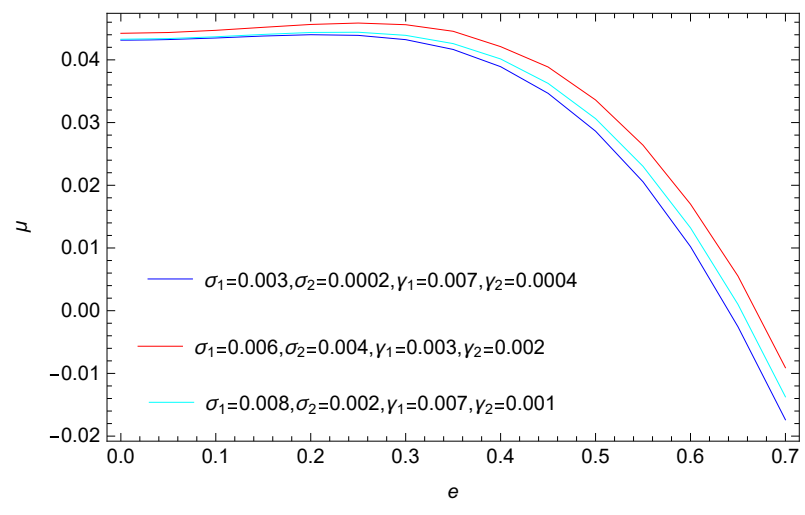

Fig. 6. The critical mass ratio versus eccentricity for different values of the triaxial parameters. The color figure can be viewed online.

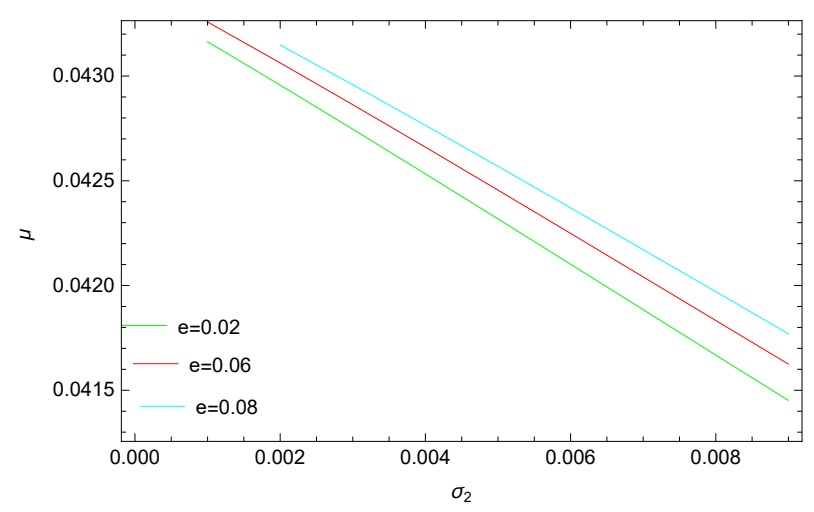

Fig. 7. The critical mass ratio versus triaxial parameter $\sigma_{2}$, for different values of the eccentricity. The color figure can be viewed online.

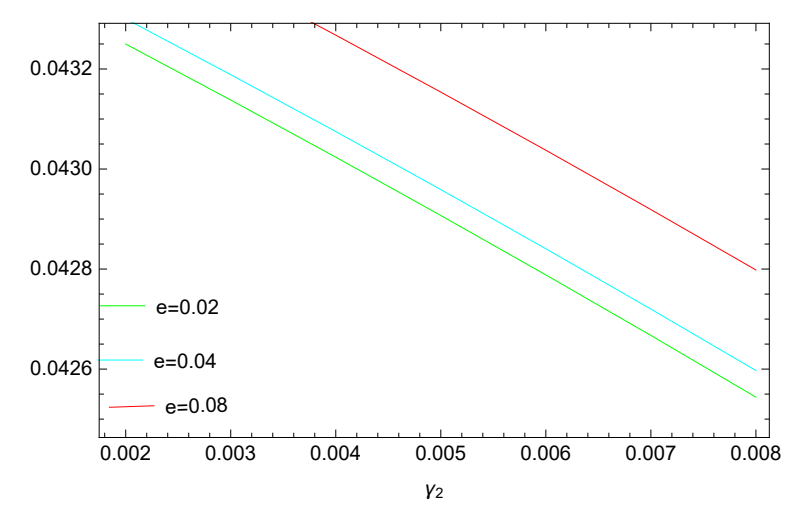

Fig. 8. The critical mass ratio versus triaxial parameter $\gamma_{2}$, for different values of the eccentricity. The color figure can be viewed online.
TABLE 1

VALUES OF THE CRITICAL MASS RATIO FOR DIFFERENT DYNAMICAL MODELS

\begin{tabular}{lllllll}
\hline$\sigma_{1}$ & \multicolumn{1}{c}{$\sigma_{2}$} & $\gamma_{1}$ & $\gamma_{2}$ & $e$ & $a$ & $\mu_{c}$ \\
\hline 0 & 0 & 0 & 0 & 0 & 1 & 0.0385209 \\
& & & & & & \\
0.006 & 0.0004 & 0.03 & 0.002 & 0.2 & 0.92 & 0.0443621 \\
0.006 & 0.0004 & 0.03 & 0.002 & 0.4 & 0.92 & 0.0401412 \\
0.006 & 0.0004 & 0.03 & 0.002 & 0.6 & 0.92 & 0.0132093 \\
& & & & & & \\
0.003 & 0.0002 & 0.007 & 0.0004 & 0.02 & 0.92 & 0.0456447 \\
0.003 & 0.0002 & 0.007 & 0.0004 & 0.04 & 0.92 & 0.0424109 \\
0.003 & 0.0002 & 0.007 & 0.0004 & 0.07 & 0.92 & 0.00909276 \\
& & & & & & \\
0.009 & 0.002 & 0.008 & 0.002 & 0.02 & 0.92 & 0.042956 \\
0.009 & 0.004 & 0.008 & 0.002 & 0.02 & 0.92 & 0.0425331 \\
0.009 & 0.008 & 0.008 & 0.002 & 0.02 & 0.92 & 0.0416678 \\
& & & & & & \\
0.006 & 0.001 & 0.008 & 0.001 & 0.08 & 0.92 & 0.0466464 \\
0.006 & 0.001 & 0.008 & 0.005 & 0.08 & 0.92 & 0.0431538 \\
0.006 & 0.001 & 0.008 & 0.008 & 0.08 & 0.92 & 0.0427982 \\
\hline
\end{tabular}

stroyed. Table 1 gives the values of the critical mass for some selected dynamical models. These values are computed under the effect of the included perturbations. We notice from the table that when the triaxiality coefficients $\left(\sigma_{i}\right.$ and $\left.\gamma_{i}\right)$ are equal to zero, $\mu_{c}$ reduces to the critical mass value of the classical restricted problem (see Figure 3). We also notice from Figures 4, 5 that the triaxiality coefficients of the primaries change the range of stability.

In addition, we can also investigate the behavior of the system by plotting the critical mass ratio against the eccentricity for different values of the triaxial parameters $\sigma_{i}$ and $\gamma_{i}$. We plot different combinations of triaxial parameters and a stable region is observed. Figure 6 shows that the increase in the eccentricity reduces the region of stability of the infinitesimal body about the triangular points. Figures 7 and 8 represent the variation of the critical mass with the triaxial parameters when the eccentricity is constant. We can see from the two figures that increasing the triaxial parameters results in a destabilizing effect in the system. 


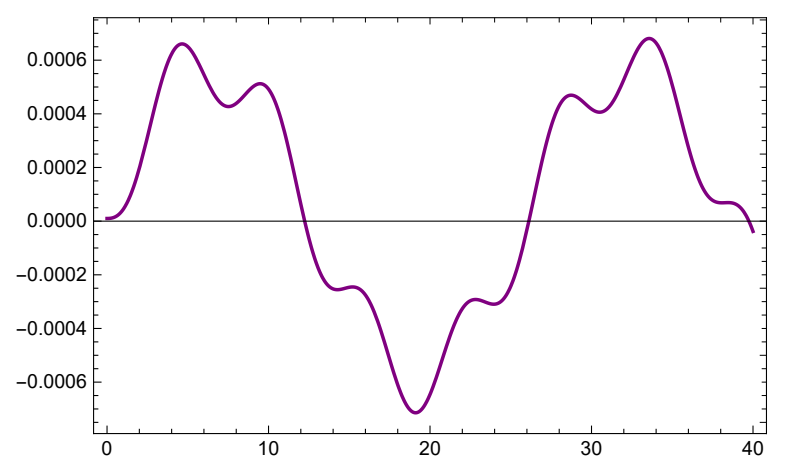

Fig. 9. $x[t]$ as a function of time with triaxiality coefficients $\sigma_{1}=0.007, \sigma_{2}=0.002, \gamma_{1}=0.005, \gamma_{2}=0.002$, $\mu=0.01, e=0.01$ and $a=0.92$. The color figure can be viewed online.

\section{MOTION IN THE PROXIMITY OF THE TRIANGULAR POINTS}

It is known that periodic orbits represent the backbone in the study of dynamical systems in celestial mechanics. Since in the range $0 \leq \mu \leq \mu_{c}$, the roots of the characteristic polynomial of the present system are purely imaginary, we conclude that the motion around the triangular points $L_{4}$ (or $L_{5}$ ) is stable and composed of two harmonic motions. The solutions for the perturbed motion about the triangular point $L_{4}$ (in the synodic reference frame) may be written as

$$
\begin{aligned}
& X[t]=\sum_{j=1}^{4} \alpha_{j} e^{\lambda_{j} t}, \\
& Y[t]=\sum_{j=1}^{4} \beta_{j} e^{\lambda_{j} t},
\end{aligned}
$$

where the terms with coefficients $\alpha_{j}$ and $\beta_{j}$ represent the short and long periodic motion. The constants $\alpha_{j}$ and $\beta_{j}$ are not independent. Applying the initial conditions to the solutions of the system these solutions may be written in the form

$$
\begin{gathered}
X[t]=5.80204 * 10^{-5} \sin (0.241582 t)- \\
1.31958 * 10^{-5} \sin (1.06221 t)+ \\
3.19255 * 10^{-5} \cos (0.241582 t)- \\
2.19255 * 10^{-5} \cos (1.06221 t) . \\
Y[t]=8.06288^{\bullet} * 10^{-5} \cos \left(0.241582^{`} t\right)- \\
7.062876^{\circ} * 10^{-5} \cos \left(1.06221^{`} t\right)- \\
3.054769^{\circ} * 10^{-5} \sin \left(0.241582^{\circ} t\right)+ \\
6.9475842 * 10^{-5} \sin (1.06221 t) .
\end{gathered}
$$

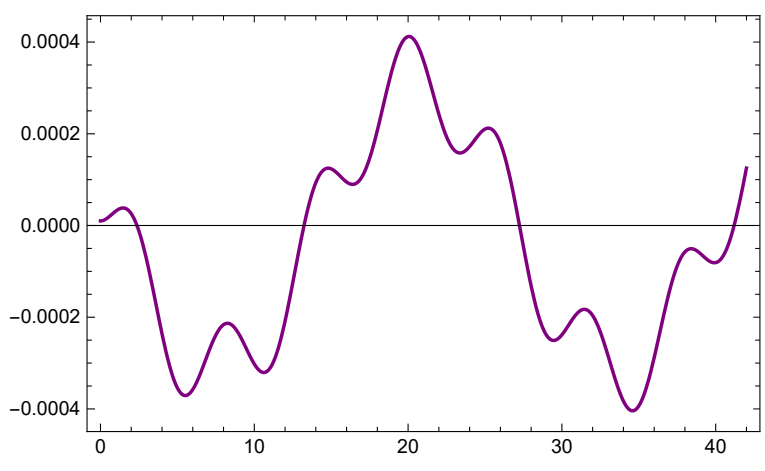

Fig. 10. $y[t]$ as a function of time with triaxiality coefficients $\sigma_{1}=0.007, \sigma_{2}=0.002, \gamma_{1}=0.005, \gamma_{2}=0.002$, $\mu=0.01, e=0.01$ and $a=0.92$. The color figure can be viewed online.

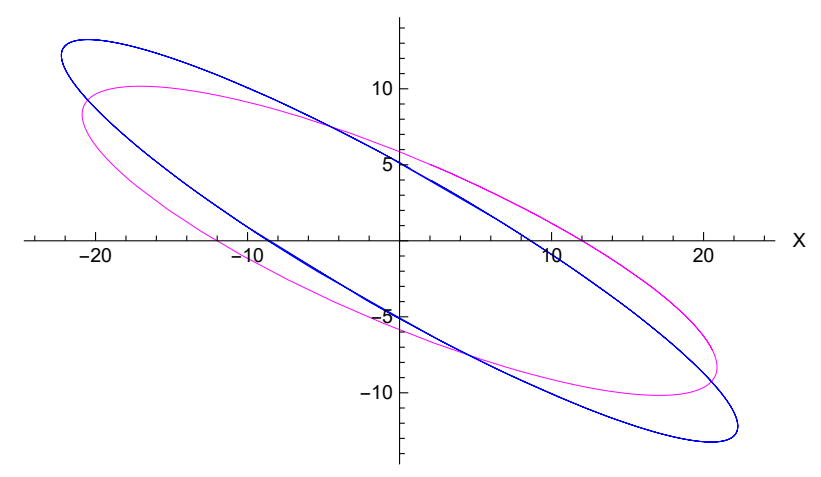

Fig. 11. Comparison between the long period motion in both the classical case (blue curve) and the present dynamical system (magenta curve). The color figure can be viewed online.

We may note from equations (29) and (30) that the solution is of the oscillatory type with fundamental periods of $\frac{2 \pi}{0.241582^{\circ}}$ and $\frac{2 \pi}{1.06221}$ times the orbital periods of the orbiting body. Hence the body will remain in the proximity of the triangular point $L_{4}$ and the motion is stable. These two different types of frequencies in the solution exist due to the fact that the resulting motion of the small body is composed of two types of motion. Firstly, a short-period motion with a period close to the orbital period of the less massive primary, and secondly a superimposed longperiod one known as a libration around the point $L_{4}$. The two solutions are depicted in Figures 9 and 10. The graph of the trajectory in the vicinity of equilibrium point $L_{4}$ is shown in Figures 11 and 12 with magenta color. The graphs contain both the short and the long-period motions. Figure 11 shows a com- 


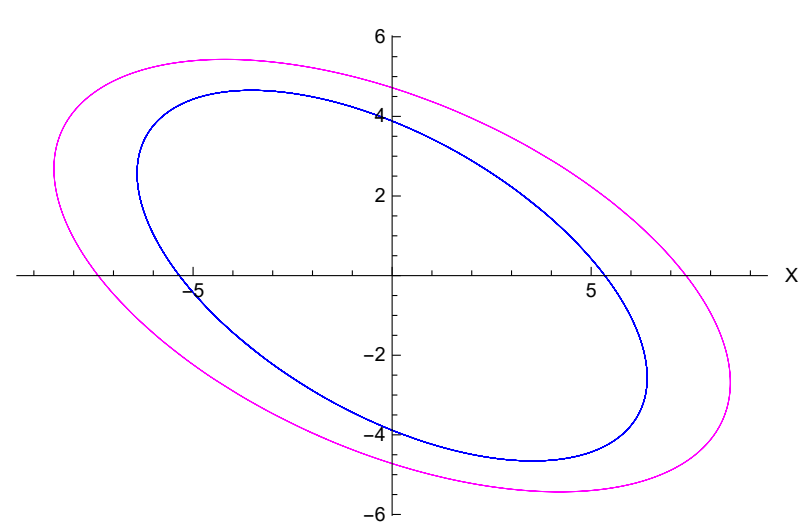

Fig. 12. Comparison between the short period motion in both the classical case (blue) and the present dynamical system (magenta). The color figure can be viewed online.

parison between the long-period motions in both the classical case (blue curve) and the present dynamical system (magenta curve), while Figure 12 shows a comparison between the two systems in the shortperiod case. It is observed that the trajectories (magenta curve) in both figures are shifted from those of the classical case. This is due to the included perturbations.

\section{CONCLUSIONS}

We have studied the existence and linear stability of the triangular points in the elliptic restricted threebody problem when the primaries are triaxial bodies. We found that the locations and the linear stability of the triangular points are affected by the triaxiality coefficients of the primaries and the eccentricity of the orbit. We also noticed that the size of the stability regions depends on the triaxiality coefficients and the eccentricity of the orbits. In addition, we studied the periodic orbits in the vicinity of the triangular points. We showed that these orbits are elliptical. Further, we observed that the shape of the periodic orbits changed due to the triaxiality of the primaries and showed a deviation from the classical case.

\section{REFERENCES}

Abd El-Salam, F. A., 2015, Ap\&SS, 357, 15 . 2019, NewA, 68, 25

Abouelmagd, Elbaz I. 2013, EM\&P, 110, 143

Ahmed, A. A., Selim, H. H., Kamel, A. K. G., et al. 2020, Applied Mathematics and Nonlinear Sciences, 5, 157

Cárcamo-Díaz, D., Palacián, J. F., Vidal, C., \& Yanguas, P. 2020, RCD, 25, 131

Kumar, P. \& Sharma, R. K. 2020, Indian Journal of Science and Technology, 13, 32

Mittal, A., Ahmad, I., \& Bhatnagar, K. B., 2009, Ap\&SS, 323,65

Musielak, Z. E. \& Quarles, B. 2014, RPPh, 77, 6, 065901

Narayan, A. \& Usha, T. 2014, Ap\&SS, 351, 135

Selim, H. H., Guirao, J. L. G., \& Abouelmagd, E. I. 2019, Discrete \& Continuous Dynamical Systems-S, 12, 703

Singh, J. \& Begha, J. M. 2011, Ap\&SS, 332, 319

Singh, J. \& Umar, A. 2012, AJ, 143, 109

Singh, J. \& Tyokyaa, R. K. 2016, EPJP, 131, 365

Szebehely, V. G. 1967, Theory of orbits. The restricted problem of three bodies (New York, NY: Academic Press)

Zahra, K., Radwan, M., \& Awad, M. E. 2016, Nonlinear Analysis and Differential Equations, 4, 609

Zahra, K., Awad, Z., Dwidar, H. R., \& Radwan, M. 2017, SerAJ, 195, 47

Nihad S. Abd El Motelp: Astronomy; Astronomy Department, National Research Institute of Astronomy and Geophysics (NRIAG), 11421 Helwan, Cairo, Egypt (nihad.saad@nriag.sci.eg).

M. Radwan: Astronomy; Space Science and Metreology Department, Faculty of Science, Cairo University, 12613 Giza, Egypt (mradwan@sci.cu.edu.eg). 